\title{
Relevant risk factors and the prognostic impact of positive resection margins after endoscopic resection of gastrointestinal neuroendocrine tumors
}

\author{
Jing Wen ${ }^{1}$, Bin Yan ${ }^{2}$, Jing Yang ${ }^{2}$, Zhongsheng Lu², Xuqiang Bian $^{3}$, Jin Huang ${ }^{3}$ \\ ${ }^{1}$ Department of Gastroenterology and Hepatology, Chinese PLA 984 Hospital, Beijing, China \\ ${ }^{2}$ Department of Gastroenterology and Hepatology, Chinese PLA General Hospital, Beijing, China \\ ${ }^{3}$ Department of Gastroenterology, Chinese PLA 988 Hospital, Zhengzhou, China
}

Videosurgery Miniinv 2020; 15 (2): 276-282

DOI: https://doi.org/10.5114/wiitm.2019.89192

\begin{abstract}
Introduction: Endoscopic resection for the treatment of gastrointestinal neuroendocrine tumors has a risk of resection margin residues. The related risk factors and prognosis of post-endoscopic resection margin residues have not been fully evaluated.

Aim: To investigate the associated risk factors and prognostic impact of resection margin residues after endoscopic resection of gastrointestinal neuroendocrine tumors.

Material and methods: We conducted a retrospective analysis of 129 patients who underwent endoscopic resection for the treatment of gastrointestinal neuroendocrine tumors. Sex, age, location, diameter of tumor, depth of invasion, endoscopic treatment methods, endoscopic ultrasonography (EUS) evaluation, and the work experience of endoscopists were evaluated as potential risk factors. In addition, the prognoses of patients with positive resection margins were analyzed.

Results: A total of 18 (18/129, 14.0\%) patients exhibited positive resection margins after endoscopic resection. Among 16 successfully followed-up patients, 1 died due to rupture of pulmonary artery aneurysms, 2 underwent supplementary surgical operations, and 2 underwent additional endoscopic submucosal dissection. The remaining 11 patients were periodically followed up, and no recurrences were found. The results of univariate analysis suggested that endoscopic treatment method, the depth of invasion, and EUS evaluation correlated with positive resection margin. Multivariate regression analysis suggested that the depth of invasion and EUS evaluation were risk factors for resection margin residues.

Conclusions: The depth of invasion and EUS evaluation are independent risk factors for positive resection margins after endoscopic resection. This finding suggests that a greater depth of invasion increases the risk for positive resection margins, while EUS evaluation before resection decreases this risk.
\end{abstract}

Key words: gastrointestinal neuroendocrine tumors, endoscopic treatment, risk factors, positive resection margins.

\section{Introduction}

With the continuous development in endoscopic resection methods, gastrointestinal neuroendocrine tumors can be detected in the early stages and treated promptly using more advanced endoscopic resection method [1-3]. Neuroendocrine tumors exhibit local infiltrative growth as submucosal lesions, whose grade of malignancy is determined based on

Address for correspondence

Jin Huang, Department of Gastroenterology, Chinese PLA 988 Hospital, Zhengzhou, China, e-mail: jinhuangdoc@163.com 
tumor size and depth of invasion [4]. This feature of invasive growth and the limitations of minimally invasive endoscopic resection often result in residues at resection margins after endoscopic treatment, which may have a direct influence on the protocol chosen for treatment and prognosis of the disease. Only a few multi-center studies with large samples have reported the final management and long-term follow-up results of patients with residues at resection margins after endoscopic treatment, while systematic statistics and analysis on factors relevant to postoperative residues at resection margins have not yet been reported.

\section{Aim}

Through factors relevant to patients, tumors, and operators, this study aimed to conduct a detailed analysis on the related risk factors and prognosis of patients with residues at resection margins after undergoing endoscopic resection of gastrointestinal neuroendocrine tumors. We aimed to further improve the curative effect of endoscopic resection of gastrointestinal neuroendocrine tumors and to provide feasible suggestions about follow-up measures for patients with positive resection margins.

\section{Material and methods}

\section{Patients}

We conducted a retrospective analysis of the clinical, endoscopic, pathological, and follow-up data of 129 patients with gastrointestinal neuroendocrine tumors who underwent endoscopic resection at our hospital's endoscopy center from December 2007 to December 2017. Patients 1) who underwent complete removal of mucosal lesions, 2) in whom gastrointestinal neuroendocrine tumor was diagnosed pathologically after endoscopic resection, 3 ) in whom computed tomography (CT) showed the absence of lymphatic metastasis, and 4) with integral records of data were included. Clinical symptoms were mainly abdominal pain, diarrhea, and abdominal distension, without carcinoid syndrome. This study was approved by the hospital's ethics committee. All patients and their relatives were informed of the risks and benefits of endoscopic resection, and written informed consent was obtained from all participants.

\section{Endoscopic treatment methods and pathological examination}

Three treatment methods under endoscopic guidance were adopted to excise a tumor: endoscopic polypectomy, endoscopic mucosal resection (EMR), and endoscopic submucosal dissection (ESD). A specimen was obtained after endoscopic resection for pathological examination; serial section examination was performed on suspected areas to determine the nature of lesions, classification, and presence or absence of vascular invasion. Positive resection margins were defined as the presence of tumor cells at the lateral and/or vertical resection margins. If it was difficult to determine whether a residue caused by coagulation was present on the basal margin, it was classified as "unable to assess", which was also regarded as residues at resection margins.

\section{Statistical analysis}

Variables such as sex, age, location of the lesion, diameter of tumor, depth of invasion, endoscopic treatment method, endoscopic ultrasonography (EUS) examination performed before resection, and the work experience of endoscopic physicians were analyzed as potential risk factors. The normally distributed data were expressed as mean \pm standard deviation (SD), while non-normally distributed data were expressed as median. The statistical analysis was performed using the $\chi^{2}$ and $U$ tests. Associations between the variables and the positive margin risk factors were examined using multivariate logistic regression models. A probability level of $p<0.05$ was set for statistical significance. Data processing was performed using the SPSS software package.

\section{Results}

\section{General data}

This study was a retrospective analysis of prospectively collected data from 129 patients with gastrointestinal neuroendocrine tumors who met the inclusion criteria; of the total study participants, 83 were men and 46 were women, with a ratio of $1.8: 1$ and mean age of $47.5 \pm 11.9$ (range: $16-80$ ) years.

\section{Residues at resection margins and follow-up data}

The surgical procedures mentioned in the study were performed by endoscopic physicians with more 
than 5 years of experience and with professional training. The mean tumor diameter was $7 \pm 3 \mathrm{~mm}$ (range: $3-18 \mathrm{~mm}$ ). Of the patients, 18 had residues at the resection margins with a positive rate of $14.0 \%$, including 3 who had residues at the lateral resection margins with a positive rate of $2.3 \%$ and 15 who had residues at the vertical resection margins with a positive rate of $11.6 \%$. There was absence of vessel invasion or submucosal lymphatic metastasis.

Eighteen patients with positive resection margins agreed to undergo supplementary surgical treatment; after communicating with patients and their family members, some refused to undergo the said operation. The patients who refused the operation would be re-examined under an endoscope 1 month later, with biopsy performed on the suspected areas; if the cells of neuroendocrine tumors are detected through biopsy, an extended resection will be performed under endoscopic guidance; however, if residues are not detected in patients who had a repeat endoscopy, they will be followed up with endoscopic evaluation within 3 months, after 6 months, and after 1 year, as well as in every year in the future, and an abdominal CT scan will be performed 6 and 12 months after the operation. The patients will no longer be followed up 5 years after the operation. Selection of the above treatment programs was based on the patients' health conditions and the choices of their family members. Eighteen patients with positive resection margins were followed up for 2 months to 5 years, and the average follow-up period was 26.4 months. Sixteen patients were successfully followed up, while two were lost to follow-up. The follow-up rate was $88.9 \%$. During the follow-up period, one patient died 1 year after the operation due to rupture of pulmonary artery aneurysm, independent of the disease. During reexaminations and follow-up visits, two patients showed stable health conditions 5 years after undergoing supplementary surgical treatment. Two patients underwent additional ESD. The remaining 11 patients did not exhibit recurrence or metastases during follow-up visits. Table I presents the specific resection margins with residues and follow-up results.

\section{Comparison between the groups with and without resection margin residues}

Several relevant factors may have influenced the status of resection margins including sex, age, lesion location, diameter of tumor, endoscopic treatment method, depth of invasion, EUS examination performed before resection, and the work experience of endoscopic physicians. Univariate and multivariate analyses were conducted to determine the relevant factors that may have influenced the resection margins following resection performed under endoscopic guidance. As shown in Table II, there were no significant differences in patients' sex, age, lesion location, diameter of tumor, or working experience of endoscopic physicians in the univariate analysis of resection margins. In contrast, endoscopic treatment method, depth of invasion, and EUS examination were risk factors for the occurrence of resection margin residues. To exclude confounding factors, using logistic regression analysis (as shown in Table III), only depth of tumor invasion and EUS examination were considered as risk factors for the occurrence of resection margin residues.

\section{Discussion}

Slow-growing gastrointestinal neuroendocrine tumors with good histological differentiation that rarely metastasize are considered as low-grade malignant tumors, which are within the confines of benign and malignant tumors, but have latent malignancy and potential for recurrence and metastasis [5]. As a minimally invasive technique, endoscopic resection may benefit patients diagnosed with gastrointestinal neuroendocrine tumors. Recently, endoscopic submucosal dissection has been adopted as treatment for digestive tract submucosal tumors and was found to be effective [6, 7]. However, a significant difference was observed in the occurrence rate of positive resection margins after endoscopic resection. Through comprehensive considerations on the three methods, the study obtained a positive incidence of $14.0 \%$. Statistical analysis on the confounding factors influenced by the working experience of endoscopic physicians was also performed, and no statistical significance was found in both univariate and multivariate regression analyses. Univariate and multivariate statistical methods were used in the study to analyze the objective and subjective relevant risk factors and conclusions made for positive resection margins after endoscopic resection of gastrointestinal neuroendocrine tumors. No similar articles related to this study have been published. 
Table I. Post-endoscopic resection residues at resection margins in patients with gastrointestinal neuroendocrine tumors and the follow-up data

\begin{tabular}{|c|c|c|c|c|c|c|}
\hline $\begin{array}{l}\text { Patient } \\
\text { no. }\end{array}$ & $\begin{array}{l}\text { Tumor } \\
\text { location }\end{array}$ & $\begin{array}{c}\text { Tumor size } \\
{[\mathrm{mm}]}\end{array}$ & Treatment technique & Tumor depth & $\begin{array}{c}\text { Margin } \\
\text { involvement }\end{array}$ & $\begin{array}{l}\text { Additional } \\
\text { treatment }\end{array}$ \\
\hline 1 & Duodenum & 9 & ESD & Muscularis & VRM & Lost to follow-up \\
\hline 2 & Duodenum & 8 & EMR & Submucosa & VRM & No \\
\hline 3 & Rectum & 6 & Endoscopic polypectomy & Submucosa & VRM & No \\
\hline 4 & Rectum & 5 & Endoscopic polypectomy & Submucosa & VRM & No \\
\hline 5 & Rectum & 8 & Endoscopic polypectomy & Submucosa & VRM & No \\
\hline 6 & Rectum & 5 & Endoscopic polypectomy & Submucosa & VRM & No \\
\hline 7 & Rectum & 6 & Endoscopic polypectomy & Muscularis & VRM & Surgery \\
\hline 8 & Rectum & 8 & Endoscopic polypectomy & Submucosa & VRM & No \\
\hline 9 & Rectum & 6 & Endoscopic polypectomy & Mucosa & LRM & Additional ESD \\
\hline 10 & Rectum & 8 & Endoscopic polypectomy & Submucosa & VRM & Additional ESD \\
\hline 11 & Rectum & 6 & Endoscopic polypectomy & Submucosa & VRM & No \\
\hline 12 & Rectum & 5 & Endoscopic polypectomy & Submucosa & LRM & No \\
\hline 13 & Rectum & 5 & EMR & Submucosa & VRM & Death \\
\hline 14 & Rectum & 6 & EMR & Submucosa & VRM & No \\
\hline 15 & Rectum & 10 & ESD & Submucosa & VRM & No \\
\hline 16 & Rectum & 8 & EMR & Muscularis & VRM & Surgery \\
\hline 17 & Rectum & 1 & EMR & Submucosa & VRM & No \\
\hline 18 & Rectum & 1 & EMR & Submucosa & LRM & Lost to follow-up \\
\hline
\end{tabular}

EMR - endoscopic mucosal resection, ESD - endoscopic submucosal dissection, VRM - vertical resection margin, LRM - lateral resection margin.

Various endoscopic resection procedures such as endoscopic polypectomy, strip biopsy, aspiration resection, and band-snare resection have been described as effective treatments for gastrointestinal neuroendocrine tumors [8-10]. However, about 75\% of the tumors extend into the submucosa [11]. Traditional endoscopic resection procedures may not provide adequate resection margins. Therefore, the incidence of resection margin residues will possibly increase. The incidence of resection margin residues reported in other literature reached up to $62 \%$ [1214]. The study provided a comparison between endoscopic polypectomy, EMR, and ESD, whose resection margin residue rates were $33.3 \%, 15.8 \%$, and $4.7 \%$ respectively, and the three methods showed significant differences. ESD has been approved for en bloc and complete resection of early gastric cancer; moreover, it enables clinicians to secure a higher negative resection margin rate than EMR and endo- scopic polypectomy. However, based on the logistic regression analysis results, endoscopic resection procedures are not independent risk factors. This finding is related to the results of the retrospective analysis, but not on the results of the randomized controlled trial. With regard to depth of tumor invasion to submucosa assessed by an ultrasonic endoscope before the operation, ESD must be performed to excise the tumors and ensure complete resection.

Some previous studies showed that the risk factors for occurrence of resection margin residues after endoscopic resection of early gastric cancer are directly related to the size of the tumors $[15,16]$. However, Isomoto et al. [17] reported that tumor size had no significant impact on curative resection, and our study failed to make corresponding conclusions. Restrained tumor growth was among the indications for endoscopic resection of neuroendocrine tumors. Patients with tumors less than $10 \mathrm{~mm}$ in diameter 
Table II. Comparison of the groups with and without post-endoscopic resection residues at resection margins

\begin{tabular}{|c|c|c|c|}
\hline Parameter & $\begin{array}{l}\text { Residues at } \\
\text { resection } \\
\text { margin } \\
(n=18)\end{array}$ & $\begin{array}{l}\text { No residue } \\
\text { at resection } \\
\text { margin } \\
(n=111)\end{array}$ & $P$-value \\
\hline Age [years] & $45.7 \pm 11.3$ & $47.8 \pm 11.9$ & 0.48 \\
\hline \multicolumn{4}{|l|}{ Sex: } \\
\hline Female & $5(22.7 \%)$ & $41(39.6 \%)$ & 0.63 \\
\hline Male & $13(15.7 \%)$ & $70(84.3 \%)$ & \\
\hline Location: & & & 0.11 \\
\hline Stomach & 0 & 8 & \\
\hline Duodenum & 2 & 2 & \\
\hline Sigmoid & 0 & 3 & \\
\hline Rectum & 16 & 98 & \\
\hline $\begin{array}{l}\text { Diameter of tumor } \\
{[\mathrm{mm}]}\end{array}$ & $6.9 \pm 3.2$ & $7.2 \pm 1.8$ & 0.77 \\
\hline \multicolumn{3}{|c|}{ Endoscopic treatment methods: } & 0.02 \\
\hline $\begin{array}{l}\text { Endoscopic } \\
\text { polypectomy }\end{array}$ & 10 & 30 & \\
\hline EMR & 6 & 38 & \\
\hline ESD & 2 & 43 & \\
\hline \multicolumn{3}{|c|}{ EUS evaluation before resection: } & 0.01 \\
\hline Yes & 10 & 93 & \\
\hline No & 8 & 18 & \\
\hline \multicolumn{3}{|l|}{ Working experience: } & 0.11 \\
\hline More than 10 years & 7 & 69 & \\
\hline Less than 9 years & 11 & 42 & \\
\hline \multicolumn{3}{|l|}{ Depth of invasion: } & $<0.001$ \\
\hline Mucous layer & 1 & 53 & \\
\hline Submucosal layer & 15 & 58 & \\
\hline Muscularis & 2 & 0 & \\
\hline
\end{tabular}

are indicated for endoscopic resection if the tumor does not invade the muscular layer, has no ulcer formation, or has no hollow surface [18]. However, due to the advances in EMR and ESD technology, for tumors with a diameter of less than $20 \mathrm{~mm}$, endoscopic resection is always selected $[19,20]$.

To prevent the occurrence of residues in lateral resection margins, it is necessary to perform EUS before the operation to correctly measure the tumor diameter and determine the scope of lesions. The study showed that EUS examination before endoscopic resection was an independent risk factor for the occurrence of resection margin residues after the operation. EUS was found to be useful for measuring the size and depth of gastrointestinal neuroendocrine tumors, which is essential for determining appropriate treatment [21, 22]. EUS should be used to assess lesion scope and depth of invasion as well as the possibility for lymphatic metastasis before the operation, which is crucial to reduce the risk of resection margin residues and increase the curative resection rate.

Depth of invasion is an independent risk factor for the occurrence of resection margin residues, which is undoubted. Determining the depth of invasion is important for preventing the occurrence of resection margin residues, is performed before the operation, and avoids unnecessary surgical treatments that may lead to the occurrence of residues at vertical resection margins; it is necessary to reduce the thickness of the submucosal layer while avoiding excessive thermal coagulation injury into the mucosa.

There are no established guidelines on whether a surgical operation should be actively performed in patients with resection margin residues after endoscopic resection of gastrointestinal neuroendocrine tumors. Among 16 patients with resection margin residues studied by Lee et al. [20], only 4 underwent supplementary treatment; the remaining 12 were followed up after the operation and had no recurrence or metastasis. The follow-up period lasted for 38 months. Based on the data of this group, 18 patients had resection margin residues. Of them, two were lost to follow-up, two agreed to undergo surgical treatment, two agreed to undergo additional ESD, and one died from other disease. The other 11 patients did not develop recurrence or metastasis during the follow-up visit.

For tumors that occur in other locations, such as the duodenum, surgical difficulties and risks may be increased; if the tumor develops in the distal rectum, the anus will also be removed, which decreases patients' quality of life. In addition, the old and the weak are unable to tolerate the operation. Following the patients' will and considering their health conditions, a frequent follow-up must be conducted. During follow-up, it is necessary to perform a pathological biopsy for assessment and a CT scan. However, the risk for distant metastasis is high in patients 
Table III. Results of multivariate logistic regression analysis

\begin{tabular}{|lccc|}
\hline Parameter & $P$-value & OR & $95 \% \mathrm{Cl}$ \\
\hline Sex & 0.21 & 0.30 & $0.05-1.97$ \\
\hline Age & 0.50 & 0.98 & $0.92-1.04$ \\
\hline $\begin{array}{l}\text { Diameter } \\
\text { of tumor }\end{array}$ & 0.95 & 1.07 & $0.10-11.25$ \\
\hline Location & 0.48 & 0.20 & $0.00-17.21$ \\
\hline $\begin{array}{l}\text { Endoscopic resec- } \\
\text { tion procedures }\end{array}$ & 0.94 & 1.04 & $0.35-3.08$ \\
\hline $\begin{array}{l}\text { Depth of invasion } \\
\text { Perform EUS } \\
\text { before resection }\end{array}$ & 0.00 & 537.64 & $23.52-12291.88$ \\
\hline Work experience & 0.13 & 0.20 & $0.03-1.59$ \\
\hline
\end{tabular}

with 10-20-mm tumors that extend beyond the submucosa and even infiltrate into the muscularis propria [23]; therefore, additional surgical intervention may be warranted.

Our study had some limitations. This was a single-center retrospective study; thus, bias factors could not be eliminated. To sum up, the study analyzed the relevant risk factors of resection margin residues and patients' prognosis; based on the results of data analysis, it is necessary to use EUS in order to assess the size and depth of tumor invasion before the operation, to determine the depth of lesion invasion, to identify the indications for surgical treatment under endoscopic guidance, and to perform ESD as an additional treatment, which can guard against resection margin residues and prevent recurrence after the operation.

\section{Conclusions}

The ESD has been approved as an endoscopic treatment for gastrointestinal neuroendocrine tumors; moreover, it can ensure a higher negative resection margin rate than EMR and endoscopic polypectomy. Patients with positive margins have lower incidence of recurrence and metastasis of gastrointestinal neuroendocrine tumors. Extended surgical resection may be avoided with close follow-up and observation.

\section{Acknowledgments}

Jing Wen and Bin Yan contributed equally to this work. This study was supported by the Military Med- ical Science and Technology Youth Training Project (No. 13QNP034).

\section{Conflict of interest}

The authors declare no conflict of interest.

\section{References}

1. Bertani E, Ravizza D, Milione M, et al. Neuroendocrine neoplasms of rectum: a management update. Cancer Treat Rev 2018; 66: 45-55.

2. Maasberg S, Jürgensen C, Scheerer F, et al. Endoscopic therapy for gastroduodenal neuroendocrine neoplasms. Chirurg 2016; 87: 271-9.

3. Eick J, Steinberg J, Schwertner C, et al. Rectal neuroendocrine tumors: endoscopic therapy. Chirurg 2016; 87: 288-91.

4. Kinoshita T, Kanehira E, Omura K, et al. Transanal endoscopic microsurgery in the treatment of rectal carcinoid tumor. Surg Endosc 2007; 21: 970-4.

5. Modlin IM, Lye KD, Kidd M. A 5-decade analysis of 13,715 carcinoid tumors. Cancer 2003; 97: 934-59.

6. Ebi M, Nakagawa S, Yamaguchi Y, et al. Endoscopic submucosal resection with an endoscopic variceal ligation device for the treatment of rectal neuroendocrine tumors. Int I Colorectal Dis 2018; 33: 1703-8.

7. Takita M, Sakai E, Nakao T, et al. Clinical outcomes of patients with small rectal neuroendocrine tumors treated using endoscopic submucosal resection with a ligation device. Digestion 2019; 99: 72-8.

8. Fujimura Y, Mizuno M, Takeda M, et al. A carcinoid tumor of the rectum removed by strip biopsy. Endoscopy 1993; 25: 428-30.

9. Kobayashi K, Katsumata T, Yoshizawa S, et al. Indications of endoscopic polypectomy for rectal carcinoid tumors and clinical usefulness of endoscopic ultrasonography. Dis Colon Rectum 2005; 48: 285-91.

10. Jung IS, Ryu CB, Kim JO, et al. Rectal carcinoid treated by EMR. Gastrointest Endosc 2003; 58: 253.

11. Matsumoto T, lida M, Suekane H, et al. Endoscopic ultrasonography in rectal carcinoid tumors: contribution to selection of therapy. Gastrointest Endosc 1991; 37: 539-42.

12. Ono A, Fujii T, Saito Y, et al. Endoscopic submucosal resection of rectal carcinoid tumors with a ligation device. Gastrointest Endosc 2003; 57: 583-7.

13. Nagai T, Torishima R, Nakashima H, et al. Saline-assisted endoscopic resection of rectal carcinoids: cap aspiration method versus simple snare resection. Endoscopy 2004; 36: 202-5.

14. Oshitani N, Hamasaki N, Sawa Y, et al. Endoscopic resection of small rectal carcinoid tumours using an aspiration method with a transparent overcap. J Int Med Res 2000; 28: 241-6.

15. Lee JH, Lee JH, Kim KM, et al. Clinicopathological factors of multiple lateral margin involvement after endoscopic submucosal dissection for early gastric cancer. Surg Endosc 2015; 29: 3460-8.

16. Yoon H, Kim SG, Choi J, et al. Risk factors of residual or recurrent tumor in patients with a tumor-positive resection margin after endoscopic resection of early gastric cancer. Surg Endosc 2013; 27: 1561-8. 
17. Isomoto H, Shikuwa S, Yamaguchi N, et al. Endoscopic submucosal dissection for early gastric cancer: a large-scale feasibility study. Gut 2009; 58: 331-6.

18. Kiyonori K, Tomoe K, Shigeru Y, et al. Indications of endoscopic polypectomy for rectal carcinoid tumors and clinical usefulness of endoscopic ultrasonography. Dis Colon Rectum 2005; 48: 285-91.

19. Park HW, Byeon JS, Park YS, et al. Endoscopic submucosal dissection for treatment of rectal carcinoid. Gastrointest Endosc 2010; 72: 143-9.

20. Lee DS, Jeon SW, Park SY, et al. The feasibility of endoscopic submucosal dissection for rectal carcinoid tumors: comparison with endoscopic mucosal resection. Endoscopy 2010; 42 647-51.

21. Ishii N, Horiki N, Itoh T, et al. Endoscopic submucosal dissection and preoperative assessment with endoscopic ultrasonography for the treatment of rectal carcinoid tumors. Surg Endosc 2010; 24: 1413-9.

22. Zhou FR, Huang LY, Wu CR. Endoscopic mucosal resection for rectal carcinoids under micro-probe ultrasound guidance. World J Gastroenterol 2013; 19: 2555-9.

23. Jetmore AB, Ray JE, Gathright JB Jr, et al. Rectal carcinoids: the most frequent carcinoid tumor. Dis Colon Rectum 1992; 35: $717-25$

Received: 11.07.2019, accepted: 22.09.2019. 